\title{
Bombas de circulação usadas em Aquariofilia: critério de seleção com base na ecoeficiência
}

\section{Fabio Soares Arantes e Walter Cardoso Satyro*}

Programa de Pós-Graduação em Engenharia de Produção. Universidade Nove de Julho. Rua Vergueiro, 235/249. Liberdade. São Paulo-SP, Brasil (CEP 01525-000). *E-mail: satyro.walter@gmail.com.

Resumo. Apesar do crescente interesse pela Aquariofilia, escassos são os artigos científicos que contribuem com uma abordagem mais técnica, com respeito aos equipamentos nela empregados. 0 presente estudo tem por objetivo o desenvolvimento de um critério de seleção de bombas de circulação usadas para Aquariofilia baseado na ecoeficiência. A metodologia empregada foi a pesquisa de campo, através de questionário estruturado, aplicado pessoalmente a 30 especialistas do setor de Aquarismo. Os resultados mostraram que os requisitos mais importantes foram que as bombas devem ter baixo preço e boa qualidade. Isto pode fazer com que uma bomba de baixo preço de aquisição e, nível de qualidade aceita pelo consumidor, tenha um custo total elevado, por gastar mais energia para o seu desempenho, onerando o consumidor e utilizando mais recursos naturais para o seu funcionamento, comprometendo a ecoeficiência. Este artigo tem duas contribuições científicas, com relação às bombas de circulação utilizadas em Aquariofilia, a proposição da classificação conforme suas vazões e de critério de seleção, baseado na ecoeficiência.

Palavras-chave: Ecoeficiência; Bombas d'água; Bombas de circulação; Aquarismo.

Abstract. Circulation pumps used in aquarism: Selection criteria based on eco-efficiency. Despite the growing interest in aquarism, there are few scientific papers that contribute to a more technical approach, with respect to the equipment used in it. The present study aims to develop a selection criterion for circulation pumps used for aquarism based on eco-efficiency. The methodology used was field research, through a structured questionnaire applied personally to 30 specialists in the aquarium sector. The results showed that the most important requirements were that the pumps must be low priced and of good quality. This can result in a pump with a low purchase price, and at a level of quality accepted by the consumer, having a high total cost, by spending more energy for its performance, burdening the consumer and using more natural resources for its operation, compromising eco-efficiency. This paper has two scientific contributions, in relation to the circulation pumps used

Recebido

$17 / 12 / 2020$

Aceito

30/03/2021

Disponível on line

12/04/2021

Publicado

30/04/2021

Acesso aberto

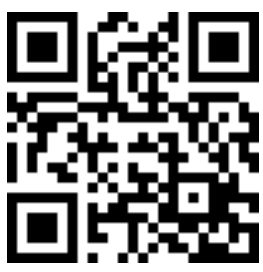

ORCID

(D) 0000-0002-9899-1812 Fabio Soares Arantes

(D) 0000-0002-0201-222X Walter Cardoso Satyro

ISSN 2359-1412/RBGAS-2020-0093/2021/8/18/10/167

Rev. Bras. Gest. Amb. Sustent.

http://revista.ecogestaobrasil.net 
in aquarium, the proposition of a classification according to their flow rates, and selection criteria based on eco-efficiency.

Keywords: Eco-efficiency; Water pumps; Circulation pumps; Aquarism.

\section{Introdução}

A preocupação em produzir de forma sustentável está cada vez mais presente nos mais variados setores da sociedade. Segundo a Comissão Brundtland (WCED, 1987), o desenvolvimento sustentável deve atender às necessidades da geração atual sem comprometer necessidades das gerações futuras.

A preocupação em preservar o meio ambiente começou a afetar vários governos e uma grande parcela da população, que começam a exercer uma pressão constante sobre as empresas e suas práticas de fabricação e de prestação de serviços (Carino et al., 2020). Assim o lucro deixa de ser o objetivo único a ser alcançado, e a crescente homogeneização de itens e serviços oferecidos ao consumidor faz com que a empresa busque obter uma figura responsável perante o mercado, figura está relacionada a certa preocupação com padrões éticos comportamentais e com a preservação ambiental (Bertheau, 2020).

A ecoeficiência pode ser conceituada como uma meta geral de criação de valor enquanto reduz o impacto ambiental e empiricamente se referindo entre a relação do impacto ambiental e custo econômico que leva a deparar com quatro tipos básicos de resultado em ecoeficiência: a intensidade ambiental, produtividade no campo do ecossistema e criação de valor, meio ambiente e custos e melhoria ambiental na eficácia de custo em indicadores de melhoria ambiental (Huppes et al., 2005; Yang et al., 2019).

A Aquicultura é definida como a produção racional, em cativeiro ou outro meio controlado, de espécies de habitat predominantemente aquático, sejam elas animais ou vegetais, tais como peixes, camarões, rãs, algas, entre outras espécies, em qualquer de seus estágios de desenvolvimento (Valenti, 2002). Ou seja, aquicultura é a produção animal ou vegetal realizada em cativeiro em ambiente aquático.

A criação de organismos aquáticos para fins ornamentais destaca-se como uma das vertentes mais promissoras da Aquicultura, cuja difusão ocorre através do uso de aquários, criações, reproduções e até mesmo a comercialização de espécies aquáticas, empregando a atividade o termo de Aquariofilia ou Aquarismo, conforme definição do Ministério da Pesca e Aquicultura (MPA, 2014).

Segundo dados da FAO (2018), através do relatório State of the World Fisheries and Aquaculture (Sofia), atualmente, o Aquarismo é uma área comercial muito vasta e influente no mercado de peixes. No entanto, a relação da maneira como esses animais são coletados está diretamente ligada à baixa remuneração dos pescadores, que expõem suas vidas com o uso de métodos ineficientes do ponto de vista qualitativo. 0 número de espécies marinhas é alto, porém a maioria dos animais capturados é descartada provocada por acidentes ou mortes destes animais, devido a falhas no processo de captura, tornando a prática de marketing insustentável o que pode levar ao esgotamento, a médio e longo prazo, das reservas naturais da biota aquática (Wood, 2001; Coe e Araújo, 2009; Lima, 2012).

Nos dias atuais, ter um aquário passou a ser entendido como uma forma de recriar um espaço da natureza dentro de casa, com finalidades educativas, recreativas e de preservação, onde se busca reproduzir, o mais fielmente possível, o habitat de origem da espécie criada (SEBRAE, 2019).

Dado o grau do desenvolvimento do mercado de aquários, é possível encontrar equipamentos modernos a preços acessíveis que ajudam a manter a qualidade da água do 
aquário, exigindo apenas cerca de meia hora de dedicação por semana, na manutenção dos equipamentos e troca de parte da água, salvando os proprietários de peixes do trabalho de limpeza de pedras, vidro e acessórios, como se fazia há algum tempo.

Com estes equipamentos modernos, evolução nas técnicas de produção, manutenção de peixes e ainda melhoramento das rações utilizadas, observa-se atualmente um aumento significativo na expectativa de vida dos animais mantidos em aquário (Colpani, 2016).

De forma geral, tanto para a aquicultura quanto para o Aquarismo o sistema de circulação de agua, atribui grande importância ao design do tanque porque otimizam energia por não ter ângulos retos, permite o acompanhamento dos movimentos de rotação e translação da terra ampliando a potência e pressão da bomba que permite a circulação e oxigenação (Ono e Kubitza, 2003).

As bombas podem ser classificadas em dinâmicas e de deslocamento positivo. Nas bombas de tipo dinâmico sua ação motriz é exclusivamente rotacional. Seu componente essencial de transmissão da energia é o impulsor ou rotor e seu princípio de funcionamento é regido pelas equações de Euler (Fox, 2004).

A bomba de circulação é classificada como bomba dinâmica, de fluxo misto, sendo um equipamento que pode ser usado para a transferência de líquidos frios e quentes, bem como para a adaptação de diversos tipos de circulação e recirculação (Fox, 2004).

Critérios de seleção de bombas são encontrados em livros de mecânica de fluidos, para bombas centrífugas utilizando água como fluido. Para tal, leva-se em consideração a vazão de bombeamento (Q) e a altura a ser vencida pela bomba $(H)$ (Pritchard e Leylegian, 2011).

Os diversos fabricantes apresentam em gráficos as curvas de suas bombas, denominada curva característica da bomba, de forma tal que entrando-se com a vazão e altura de recalque $(\mathrm{H})$ seja possível selecionar a bomba mais adequada (Fox et al., 2006; Pritchard e Leylegian, 2011).

Ono e Kubitza (2002) cita que os principais fatores que devem ser considerados no momento da seleção de uma bomba são as propriedades físicas e químicas da água (temperatura, acidez, salinidade, turbidez, quantidades de sólidos minerais), vazão demanda do projeto, altura de sucção da bomba, perda da carga na captação, que depende do tipo, diâmetro e o comprimento do tubo, bem como os tipos de conexões.

No caso de bombas de circulação este critério de seleção não é adequado, posto que o importante é a circulação do fluido, com vazão constante e, não sua altura de recalque. Não se encontrou na literatura científica, critério que pudesse auxiliar na seleção bombas de circulação utilizadas em Aquarismo, um gap de pesquisa, que este artigo busca responder.

\section{Material e método}

Este trabalho foi realizado através de pesquisa bibliográfica e pesquisa de campo realizada em com especialistas do ramo de Aquariofilia para comparar, avaliar e mensurar os critérios de seleção de bombas de circulação utilizadas atualmente e propor um critério de seleção de bombas de circulação que leve em consideração algumas ações voltadas à ecoeficiência.

Para fins deste estudo, tomou-se para exemplo seis bombas de circulação disponíveis no mercado brasileiro, quando para fins de comparação foram definidas três faixas distintas de vazão, vindo-se a classificar duas em cada uma destas faixas de vazão.

A amostra foi formada por especialistas no ramo, gerentes experientes e proprietários de tradicionais lojas do ramo de Aquariofilia, em um total de 30 especialistas, sendo delimitada a pesquisa na região metropolitana de São Paulo, também conhecida como Grande São Paulo. Dado se a maior região metropolitana do Brasil, com 
cerca de 21,5 milhões de habitantes, e uma das dez regiões metropolitanas mais populosas do mundo (IBGE, 2018). Tratando-se de uma região de interesse para a pesquisa, por ser uma das áreas de mercado no segmento da Aquariofilia mais desenvolvidas do país.

\section{Coleta de dados}

Segundo Minayo et al. (2002), para a sondagem de dados para pesquisas de campo devem ser utilizadas múltiplas fontes de evidências.

Foi dada importância no estudo para as bombas de circulação com vazões de 300 $\mathrm{L} / \mathrm{h}, 7.000 \mathrm{~L} / \mathrm{h}, 20.000 \mathrm{~L} / \mathrm{h}$, por serem faixas de vazões de maiores vendas no setor varejista, porém no mercado encontramos bombas de vazões diversas com referências às de $60 \mathrm{~L} / \mathrm{h}$ até $30.000 \mathrm{~L} / \mathrm{h}$.

Inicialmente foi realizada uma pesquisa de campo com 30 especialistas com perguntas de múltipla escolha identificando principais requisitos de maior importância nas formas utilizadas atualmente para escolha de uma bomba de circulação utilizada na Aquariofilia. Após dois meses foi aplicado, à mesma amostra da população em questão, um segundo questionário com questões estruturado com o intuito de avaliar e mensurar indicadores que pudessem indicar ações que ajudassem as empresas a se tornarem ecoeficientes.

\section{Resultados e discussão}

No primeiro questionário, $29 \%$ dos varejistas indicaram que o principal quesito levado em consideração no momento da aquisição de uma bomba de circulação é o fato do equipamento ser importado. Outros $28 \%$ informaram ser o preço de aquisição do equipamento. A qualidade foi um fator citado por $18 \%$ dos especialistas respondentes e $17 \%$ citou a fabricação nacional dos equipamentos. 0 consumo energético das bombas de circulação foi um fator lembrado por apenas $8 \%$ dos varejistas entrevistados.

Após a aplicação do primeiro questionário, foi realizada uma análise de Pareto sobre os resultados e conseguiu-se identificar que a fabricação por um produtor nacional e o consumo energético não são fatores relevantes para a escolha de uma bomba pelo setor varejista. A Figura 1 apresenta os resultados da Análise de Pareto a partir da aplicação do questionário 1.

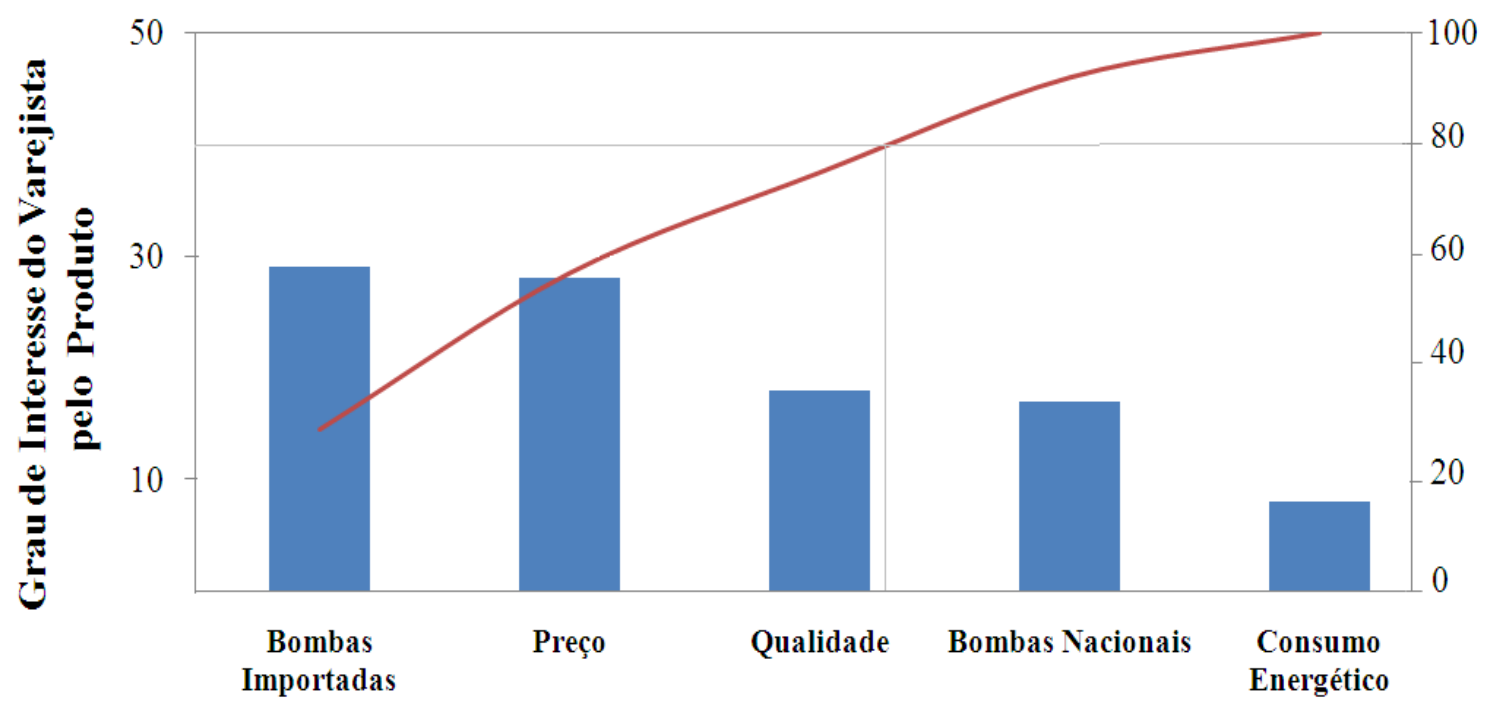

Figura 1. Analise de Pareto. 
Segue abaixo, na Tabela 1 as questões do segundo questionário.

Tabela 1. Pesquisa de campo - Questionário 2.

\begin{tabular}{|c|c|c|}
\hline Pergunta & Respostas & $\begin{array}{l}\text { Valor da } \\
\text { Resposta }\end{array}$ \\
\hline \multirow{3}{*}{$\begin{array}{l}\text { 1- Sua empresa faz o acompanhamento dos } \\
\text { volumes de água utilizados em seu sistema? }\end{array}$} & A- Nunca & 1 \\
\hline & B-Sempre, de forma periódica. & 2 \\
\hline & C- Às vezes. & 3 \\
\hline \multirow{3}{*}{$\begin{array}{l}\text { 2- Sua empresa implementa ou já implementou } \\
\text { ações para a diminuição de gasto de energia? }\end{array}$} & A- Sim, de forma periódica. & 3 \\
\hline & B- Sim, já foi implementado. & 2 \\
\hline & C- Nunca foi feito. & 1 \\
\hline \multirow{3}{*}{$\begin{array}{l}\text { 3- Em algum momento cogitou-se da } \\
\text { possibilidade de a empresa trocar as bombas } \\
\text { convencionais por econômicas do sistema? }\end{array}$} & A-Nunca & 1 \\
\hline & $\begin{array}{l}\text { B-Já fizemos estudo, mas não é } \\
\text { viável no momento. }\end{array}$ & 2 \\
\hline & C- Já fizemos as trocas & 3 \\
\hline \multirow{3}{*}{$\begin{array}{l}\text { 4- São implementadas ações para diminuir o } \\
\text { consumo de energia? }\end{array}$} & A- Nunca & 1 \\
\hline & B- Sempre, de forma periódica. & 2 \\
\hline & C- Foi feito no passado. & 3 \\
\hline \multirow{3}{*}{$\begin{array}{l}\text { 5- Existe treinamentos de funcionários nas } \\
\text { questões ambientais para o oferecimento de } \\
\text { produtos no setor varejista? }\end{array}$} & $\begin{array}{l}\text { A-Sempre, para todos os } \\
\text { funcionários. }\end{array}$ & 3 \\
\hline & $\begin{array}{l}\text { B- Somente para os funcionários } \\
\text { técnicos. }\end{array}$ & 2 \\
\hline & C-Nunca fizemos. & 1 \\
\hline \multirow{3}{*}{ 6- Sua empresa tem licença ambiental? } & $\begin{array}{l}\text { A- Sim, e dentro do prazo de } \\
\text { validade. }\end{array}$ & 3 \\
\hline & $\begin{array}{l}\text { B- Sim, mais fora do prazo de } \\
\text { validade. }\end{array}$ & 2 \\
\hline & C- Não sei/ Nunca tivemos. & 1 \\
\hline \multirow{3}{*}{$\begin{array}{l}\text { 7- Quem verifica se a empresa está cumprindo a } \\
\text { legislação ambiental? }\end{array}$} & A- Nosso contador/Advogado. & 2 \\
\hline & $\begin{array}{l}\text { B-Um especialista em meio } \\
\text { ambiente. }\end{array}$ & 3 \\
\hline & C- Não verificamos & 1 \\
\hline \multirow{3}{*}{$\begin{array}{l}\text { 8- A empresa tem programa de } \\
\text { reutilização/reciclagem de equipamentos com } \\
\text { defeitos? }\end{array}$} & $\begin{array}{l}\text { A- Sim, separamos todo o lixo e } \\
\text { vendemos. }\end{array}$ & 2 \\
\hline & B- Não temos nenhum programa. & 1 \\
\hline & $\begin{array}{l}\text { C- Sim, todos os resíduos são } \\
\text { destinados a eco pontos. }\end{array}$ & 3 \\
\hline
\end{tabular}

Fonte: Adaptado de Maroun e Schaeffer (2012).

No segundo questionário aplicado, avaliando a situação dos especialistas, em associação às práticas de ecoeficiência, os resultados demonstraram que a pontuação obtida pelos mesmos variou entre 12 e 21 pontos, de um máximo possível de 36 pontos, conforme Tabela 2. 
Tabela 2. Avaliação dos resultados

\begin{tabular}{|l|l|}
\hline Total de pontos & Resultados \\
\hline 31 a 36 & A empresa está no caminho certo para o alcance da ecoeficiência. \\
\hline 21 a 30 & $\begin{array}{l}\text { A empresa já adota algumas medidas importantes para o alcance da } \\
\text { ecoeficiência. }\end{array}$ \\
\hline 12 a 20 & É necessário dar mais atenção às questões ambientais na empresa. \\
\hline
\end{tabular}

Dos trinta especialistas pesquisados, vinte e sete ( $90 \%$ da amostra) tiveram uma pontuação entre 12 e 20 pontos, demonstrando que ainda não dão suficiente atenção às questões ambientais. Três especialistas $(10 \%$ da amostra obtiveram pontuação superior a 20 pontos, demonstrando que esses especialistas já adotam algumas medidas importantes para o alcance da ecoeficiência, mas está ainda não é amplamente incorporada nas práticas dos mesmos. Nenhum especialistas obteve uma pontuação superior a 30 , que é o patamar esperado para uma organização que de fato incorpora a ecoeficiência como uma prática de negócios. A Figura 2 a seguir apresenta a distribuição dos especialistas segundo esse critério.

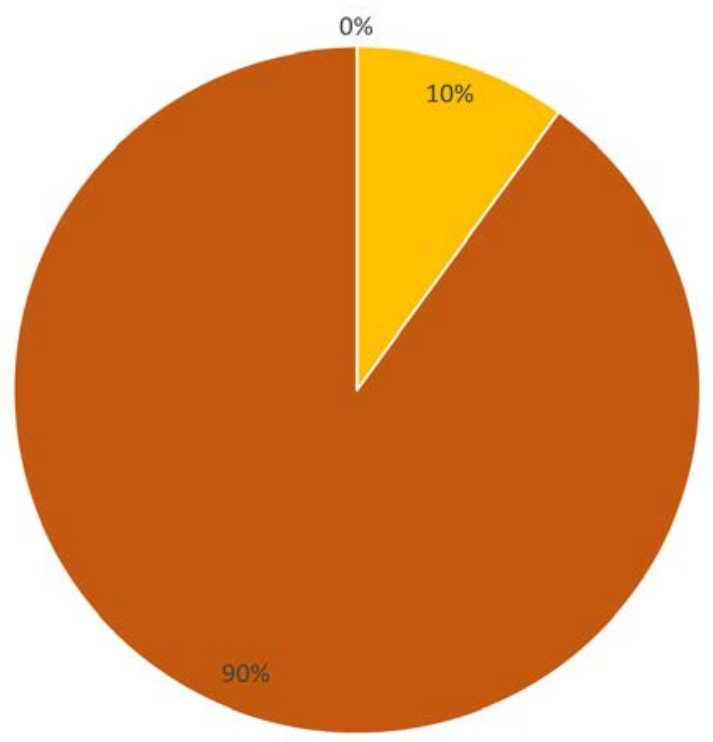

- A empresa está no caminho certo para o alcance da ecoeficiência.

- A empresa já adota algumas medidas importantes para o alcance da ecoeficiência.

- É necessário dar mais atenção às questões ambientais na empresa.

Figura 2. Resultado do segundo questionário.

Esse resultado confirma o pouco interesse que os especialistas mostraram em relação ao consumo energético das bombas de circulação, confirmando os achados do questionário 1.

Desta forma procurou-se apresentar aos especialistas, como o critério da ecoeficiência deve ser considerado como forma de seleção de bombas de circulação, ao considerar-se o consumo de energia. Assim, o valor mais alto investido em uma bomba de maior eficiência pode ser compensado por um menor consumo mensal de energia, conforme o valor monetário do consumo mensal de energia elétrica, calculado segundo a equação elaborada a partir da Elektro (2012). 


\section{Consumo [R \$ $/$ mês] $=$ POTÊNCIA[W] $/ 1000 \times 24$ x 30 x TARIFA [R\$/kWh]}

Como exemplo para aplicação dessa equação, foram utilizadas na seleção, seis modelos de bombas de circulação, escolhidas por serem as mais comercializadas pelas lojas que formaram a amostra estudada, devido a cobertura de faixas de vazões mais comercializadas, assim sendo: (1) BS (bomba submersa) produzidas por um fabricante nacional, e as outras (2) duas das marcas RESUN e JAD, ambas bombas submersas importadas, apresentadas na Tabela 3 a seguir.

De acordo com cada região do Brasil, tem-se diferentes tarifas vigentes homologadas pela Agencia Nacional de Energia Elétrica (ANEEL, 2020). Na região metropolitana de São Paulo, foco deste estudo, o valor aproximado da tarifa de eletricidade considerado foi de $\mathrm{R} \$ 0,50 / \mathrm{kWh}$. Os valores do custo de energia expressos abaixo, se referem a $\mathrm{R} \$ /$ mês.

Tabela 3. Simulação de cálculo de consumo mensal em R \$/mês.

\begin{tabular}{|l|c|c|c|c|c|}
\hline Bombas & Vazão & $\begin{array}{c}\text { Potência } \\
\text { (W) }\end{array}$ & $\begin{array}{c}\text { Preço de } \\
\text { venda* }^{*}\end{array}$ & Total $(\mathbf{k W h}$ ) $* *$ & $\begin{array}{c}\text { Custo de energia } \\
\text { (R\$/mês) }\end{array}$ \\
\hline Bs 300 & $300 \mathrm{~L} / \mathrm{h}$ & $7 \mathrm{~W}$ & $\mathrm{R} \$ 69,24$ & $5,04 \mathrm{kWh}$ & $\mathrm{R} \$ 2,52$ \\
\hline Resun 400 & $400 \mathrm{~L} / \mathrm{h}$ & $6 \mathrm{~W}$ & $\mathrm{R} \$ 108,32$ & $4,32 \mathrm{kWh}$ & $\mathrm{R} \$ 2,16$ \\
\hline Bs Eco 7.000 & $7.000 \mathrm{~L} / \mathrm{h}$ & $57 \mathrm{~W}$ & $\mathrm{R} \$ 1.068,04$ & $41,04 \mathrm{kWh}$ & $\mathrm{R} \$ 20,52$ \\
\hline Jad Eco 6.500 & $6.500 \mathrm{~L} / \mathrm{h}$ & $50 \mathrm{~W}$ & $\mathrm{R} \$ 1.205,47$ & $36 \mathrm{kWh}$ & $\mathrm{R} \$ 18,00$ \\
\hline Bs 20.000 & $20.000 \mathrm{~L} / \mathrm{h}$ & $420 \mathrm{~W}$ & $\mathrm{R} \$ 1.438,97$ & $302,4 \mathrm{kWh}$ & $\mathrm{R} \$ 151,20$ \\
\hline Jad 20.000 & $20.000 \mathrm{~L} / \mathrm{h}$ & $200 \mathrm{~W}$ & $\mathrm{R} \$ 2.491,69$ & $144 \mathrm{kWh}$ & $\mathrm{R} \$ 72,00$ \\
\hline
\end{tabular}

*Preço de venda para o consumidor final **Considerou-se o uso contínuo por 24 horas de funcionamento por dia, 30 dias por mês, sendo o custo de operação igual ao consumo (kWh) multiplicado pela tarifa de eletricidade, no valor aproximado de R\$ 0,50/kWh (Estado de São Paulo).

De acordo com o consumo individual de cada bomba, é possível comparar os modelos de bomba, duas a duas, de acordo com a faixa de vazão de operação. Na Tabela 4 a seguir, é possível observar uma redução de consumo de energia de 14,3\% em bombas de baixa vazão, 12,3\% em bombas de média vazão e de 52,4\% em bombas de alta vazão.

Tabela 4. Redução no consumo pelo uso do modelo mais eficiente.

\begin{tabular}{|l|c|c|c|c|}
\hline Bombas & Vazão & Potência (W) & Consumo mensal & Redução no consumo \\
\hline Bs 300 & $300 \mathrm{l} / \mathrm{h}$ & $7 \mathrm{~W}$ & $5,04 \mathrm{kWh}$ & $14,3 \%$ \\
\hline Resun 400 & $400 \mathrm{l} / \mathrm{h}$ & $6 \mathrm{~W}$ & $4,32 \mathrm{kWh}$ & \\
\hline Bs Eco 7.000 & $7.000 \mathrm{l} / \mathrm{h}$ & $57 \mathrm{~W}$ & $41,04 \mathrm{kWh}$ & $12,3 \%$ \\
\hline Jad Eco 6.500 & $6.500 \mathrm{l} / \mathrm{h}$ & $50 \mathrm{~W}$ & $36 \mathrm{kWh}$ & \\
\hline Bs 20.000 & $20.000 \mathrm{l} / \mathrm{h}$ & $420 \mathrm{~W}$ & $302,4 \mathrm{kWh}$ & $52,4 \%$ \\
\hline Jad 20.000 & $20.000 \mathrm{l} / \mathrm{h}$ & $200 \mathrm{~W}$ & $144 \mathrm{kWh}$ & \\
\hline
\end{tabular}


A Tabela 5 a seguir apresenta os valores de custo de energia e economia mensal, agora em valores monetários, seguindo a tarifa de eletricidade adotada, assim como os preços de venda ao consumidor das bombas de circulação.

Tabela 5. Simulação de cálculo de consumo mensal, em R\$.

\begin{tabular}{|l|c|c|c|c|}
\hline Bombas & Potência (W) & Preço de venda & Custo de Energia (R\$/Mês) & $\begin{array}{c}\text { Economia mensal } \\
\text { (R\$/Mês) }\end{array}$ \\
\hline Bs 300 & $7 \mathrm{~W}$ & $\mathrm{R} \$ 69,24$ & $\mathrm{R} \$ 2,52$ & - \\
\hline Resun 400 & $6 \mathrm{~W}$ & $\mathrm{R} \$ 108,32$ & $\mathrm{R} \$ 2,16$ & $\mathrm{R} \$ 0,36$ \\
\hline Bs Eco 7.000 & $57 \mathrm{~W}$ & $\mathrm{R} \$ 1.068,04$ & $\mathrm{R} \$ 20,52$ & - \\
\hline Jad Eco 6.500 & $50 \mathrm{~W}$ & $\mathrm{R} \$ 1.205,47$ & $\mathrm{R} \$ 18,00$ & $\mathrm{R} \$ 2,52$ \\
\hline Bs 20.000 & $420 \mathrm{~W}$ & $\mathrm{R} \$ 1.438,97$ & $\mathrm{R} \$ 151,20$ & - \\
\hline Jad 20.000 & $200 \mathrm{~W}$ & $\mathrm{R} \$ 2.491,69$ & $\mathrm{R} \$ 72,00$ & $\mathrm{R} \$ 79,20$ \\
\hline
\end{tabular}

Conforme os dados apresentados na Tabela 6, abaixo, considerando as bombas de baixa potência, para que os dois modelos sejam equivalentes em termos de custos totais (custo de aquisição + custo de energia), são necessários 109 meses de operação, ou pouco mais de 9 anos. Esse período é encontrado realizando-se a divisão da diferença de preço entre as bombas pela economia mensal proporcionada. Dessa forma, optando-se pelo modelo mais eficiente (mais caro), teria-se o retorno do investimento realizado em pouco mais de 9 anos (109 meses). Nesse caso, a economia de energia pode-se considerar irrelevante, uma vez que tem-se a economia de apenas $1 \mathrm{~W}$ de potência, e dificilmente o consumo de energia seria um requisito a ser levado em conta para escolha por um ou outro equipamento.

Realizando cálculos análogos para as bombas das outras faixas de vazão, obtém-se que são necessários 55 meses (4 anos e 7 meses) para recuperar-se o investimento realizado na bomba de média vazão mais eficiente, e necessários 13 meses (pouco mais de um ano) para recuperar o investimento realizado na aquisição da bomba de alta vazão mais eficiente. No caso das bombas de média vazão, a economia é de $7 \mathrm{~W}$, o que percentualmente representa um ganho de $12 \%$. Já no caso das bombas de maior vazão, o ganho é mais significativo $(120 \mathrm{~W})$, representando uma economia de $52 \%$. A Tabela 6 apresentada a seguir, mostra um resumo destes resultados.

Tabela 6. Resultados de consumo de energia, sua redução e período de retorno.

\begin{tabular}{|l|c|c|c|c|c|c|}
\hline Bombas & Vazão & $\begin{array}{c}\text { Potência } \\
\text { (W) }\end{array}$ & $\begin{array}{c}\text { Consumo } \\
\text { (kWh/Mês } \\
\text { ) }\end{array}$ & $\begin{array}{c}\text { Período de } \\
\text { retorno } \\
\text { (meses) }\end{array}$ & $\begin{array}{c}\text { Consumo } \\
\text { total }\end{array}$ & $\begin{array}{c}\text { Redução } \\
\text { no } \\
\text { consumo }\end{array}$ \\
\hline BS 300 & $300 \mathrm{l} / \mathrm{h}$ & $7 \mathrm{~W}$ & $5,05 \mathrm{kWh}$ & 109 & $545,4 \mathrm{kWh}$ & \\
\hline RESUN 400 & $400 \mathrm{l} / \mathrm{h}$ & $6 \mathrm{~W}$ & $4,32 \mathrm{kWh}$ & 109 & $466,56 \mathrm{kWh}$ & $14,3 \%$ \\
\hline Bs Eco 7.000 & $7.000 \mathrm{l} / \mathrm{h}$ & $57 \mathrm{~W}$ & $41,04 \mathrm{kWh}$ & 55 & $\begin{array}{c}2.216,16 \\
\mathrm{kWh}\end{array}$ & \\
\hline Jad Eco 6.500 & $6.500 \mathrm{l} / \mathrm{h}$ & $50 \mathrm{~W}$ & $36 \mathrm{kWh}$ & 55 & $1.944 \mathrm{kWh}$ & $12,3 \%$ \\
\hline BS 20.000 & $20.000 \mathrm{l} / \mathrm{h}$ & $420 \mathrm{~W}$ & $302,4 \mathrm{kWh}$ & 13 & $\begin{array}{c}3.931,2 \\
\mathrm{kWh}\end{array}$ & \\
\hline JAD 20.000 & $20.000 \mathrm{l} / \mathrm{h}$ & $200 \mathrm{~W}$ & $144 \mathrm{kWh}$ & 13 & $1.872 \mathrm{kWh}$ & $52,4 \%$ \\
\hline
\end{tabular}


Assim, os resultados obtidos variam conforme a faixa de vazão estudada. No caso das bombas de baixa e média vazões, os períodos para retorno do investimento são consideravelmente elevados, de modo que a economia de energia proporcionada dificilmente justificaria por si só a escolha pelo modelo mais eficiente.

No caso das bombas de alta vazão, o período para retorno do investimento é menor, sendo necessário pouco mais de um ano para a equivalência. No entanto, a diferença de preços dessas bombas é relevante, podendo fazer com que o consumidor não considere a contenção do consumo de energia elétrica no momento da escolha. Talvez tenha sido este o motivo do consumo de energia não ser um requisito relevante para a escolha por um modelo ou outro, dentro de uma mesma faixa de vazão, o que foi verificado com a aplicação do questionário.

0 pouco interesse pelo consumo de energia foi apontado na pesquisa de campo, com o pouco interesse pelas bombas mais eficientes, porém mais caras. E mesmo considerando clientes de alto poder aquisitivo, as revendedoras varejistas de produtos de Aquarismo relatam preocupação principalmente com o fator preço e por serem produtos importados. Esse fator provavelmente está associado a uma melhor imagem de produtos de boa qualidade associados aos produtos importados. Já as bombas nacionais são escolhidas devido ao fator preço de venda, sem consideração pelo quesito consumo de energia.

\section{Classificação de bombas de circulação segundo a vazão}

Uma vez que não existe no mercado brasileiro uma classificação para bombas de circulação usadas em Aquariofilia, que leve em consideração suas vazões, e por não se encontrar na literatura científica sequer algo parecido, propõe-se a classificação conforme Tabela 7 a seguir, como forma de sugestão de um padrão, de acordo com os seguintes critérios de vazões.

Tabela 7. Proposição de classificação de bombas de circulação usadas na Aquariofilia, conforme vazão.

\begin{tabular}{|c|c|}
\hline Vazão & Bombas de Circulação \\
\hline Baixa & Até $5.000 \mathrm{~L} / \mathrm{h}$ \\
\hline Média & Acima de $5.000 \mathrm{~L} / \mathrm{h}$ até $10.000 \mathrm{~L} / \mathrm{h}$ \\
\hline Alta & Acima de $10.000 \mathrm{~L} / \mathrm{h}$ \\
\hline
\end{tabular}

\section{Critério de seleção de bombas de circulação baseado na ecoeficiência}

A técnica usual no mercado brasileiro é considerar que um dos critérios de seleção de bomba de circulação usada em Aquariofilia deva ser a capacidade de circular toda a água de um aquário ao menos cinco vezes a cada hora, ou seja, a cada 12 min toda a água é circulada, para aquários com água doce; e ao menos dez vezes a cada hora, ou a cada 6 min toda a água do aquário é circulada, para aquários com água salgada.

Dado que não se conseguiu localizar na literatura acadêmica critérios de seleção de bombas de circulação usada em Aquariofilia, procurou-se no mercado internacional fabricante das mesmas, para se ter um parâmetro de comparação com relação ao critério de seleção empírico encontrado no mercado nacional.

Depois de pesquisas, encontrou-se uma empresa Italiana no mercado de engenharia, estabelecida há mais de 10 anos chamada Rossmant Italy Engineering, que desenvolve bombas de circulação para Aquariofilia. 
Porém o critério de seleção deste fabricante também parte do volume de água do aquário, assim aponta ser este um critério de seleção crítico que deva ser considerado. Outrossim, o mesmo atém-se ao volume de água do aquário como variável principal, e quantas vezes este volume total precisa ser recirculado por hora, o que de certa forma guarda similaridade com o critério nacional.

Desta feita, propõem-se o seguinte critério de seleção de bombas de circulação a ser utilizado em Aquariofilia:

1) Determinação do volume do aquário

2) Seleção da água utilizada: doce ou salgada, cuja as propriedades físicas e químicas da água (temperatura, acides, salinidade, turbidez, quantidades de sólidos minerais), deve-se ater ao requisitado por cada espécie de peixe do aquário.

3) Cálculo da vazão (Q) da bomba de circulação em l/h (litros por hora). Propõemse a Tabela 8 seguinte, como seleção da vazão da bomba de circulação, para uma padronização.

Tabela 8. Volume de água do aquário e vazão da bomba de circulação padronizada.

\begin{tabular}{|c|c|c|}
\hline \multirow{2}{*}{ Volume de água do aquário (L) } & \multicolumn{2}{|c|}{ Vazão da bomba de circulação (L/h) } \\
\cline { 2 - 3 } & Água doce & Água salgada \\
\hline 10 a 20 & 100 & 200 \\
\hline 20 a 40 & 200 & 400 \\
\hline 40 a 60 & 300 & 600 \\
\hline 60 a 100 & 500 & 1.000 \\
\hline 100 a 200 & 1.000 & 2.000 \\
\hline 200 a 300 & 1.500 & 3.000 \\
\hline 300 a 400 & 2.000 & 4.000 \\
\hline 400 a 600 & 3.000 & 6.000 \\
\hline 600 a 800 & 4.000 & 8.000 \\
\hline 800 a 1000 & 5.000 & 10.000 \\
\hline 1000 a 1500 & 7.000 & 15.000 \\
\hline 1500 a 2000 & 10.000 & 20.000 \\
\hline 2000 a 3000 & 15.000 & 30.000 \\
\hline 3000 a 4500 & 22.500 & 45.000 \\
\hline 4500 a 6000 & 30.000 & 60.000 \\
\hline & \multicolumn{2}{|c|}{} \\
\hline
\end{tabular}

\section{Da seleção}

Propõem-se o seguinte critério de seleção de bombas de circulação, usada em Aquariofilia, baseado na ecoeficiência (Tabela 9).

Tabela 9. Critério de seleção de bombas de circulação, usada em Aquariofilia, baseado na ecoeficiência.

\begin{tabular}{|c|c|c|c|}
\hline \multirow{2}{*}{$\begin{array}{c}\text { Vazão da bomba de } \\
\text { circulação (vide Tabela 8) }\end{array}$} & \multicolumn{3}{|c|}{ Prioridade } \\
\hline & 19 & $2^{\mathbf{a}}$ & $3 \underline{\mathbf{a}}$ \\
\hline Baixa vazão $(<5.000 \mathrm{~L} / \mathrm{h})$ & & & \multirow{3}{*}{$\begin{array}{l}\text { Preço de } \\
\text { aquisição }\end{array}$} \\
\hline $\begin{array}{c}\text { Média vazão (entre } 5.000 \mathrm{~L} / \mathrm{h} \\
\text { e } 10.000 \mathrm{~L} / \mathrm{h} \text { ) }\end{array}$ & garantia do fabricante & energia & \\
\hline $\begin{array}{c}\text { Alta vazão } \\
(>10.000 \mathrm{~L} / \mathrm{h})\end{array}$ & $\begin{array}{l}\text { Menor consumo de } \\
\text { energia }\end{array}$ & $\begin{array}{l}\text { Maior tempo de } \\
\text { garantia do fabricante }\end{array}$ & \\
\hline
\end{tabular}


Pequenas vazões (até $5.000 \mathrm{~L} / \mathrm{h}$ ): Dado o baixo consumo energético e, longo de tempo de retorno, propõem-se adotar a bomba que apresente maior tempo de garantia, pois se o fabricante dá maior tempo de garantia supõem-se que seja produto mais durável, visando-se assim a não impactar o meio-ambiente com um produto que venha a falhar em serviço precocemente, além do risco de poder comprometer a vida dos peixes.

Médias vazões (entre $5.000 \mathrm{~L} / \mathrm{h}$ e $10.000 \mathrm{~L} / \mathrm{h}$ ): Dado o consumo energético médio e o tempo de retorno, propõem-se selecionar bomba de circulação com maior tempo de garantia, se possível associada a uma que tenha o menor consumo energético.

Altas vazões (acima de $10.000 \mathrm{~L} / \mathrm{h}$ ): utilizar a bomba de circulação de menor potência possível para uma dada vazão, por representar um menor consumo energético, reduzindo o impacto ambiental, além do capital poder ser amortizado ao longo da vida da bomba, como mostrado anteriormente, atendendo o critério da ecoeficiência.

\section{Conclusões}

Como conclusão geral deste trabalho, foi verificado que a atividade de Aquariofilia carece de uma padronização para as bombas de circulação de água usadas, com relação a uma classificação conforme suas vazões, o que pode gerar considerações de mercado que variem de um estabelecimento para outro.

Notou-se também a falta de um critério de seleção destas bombas de circulação, usadas em Aquariofilia que levasse em conta a ecoeficiência.

A Aquariofilia é um importante segmento do setor de aquicultura, representando um valor não desprezível de transações tanto em termos de importações como de exportações.

Foi realizada uma pesquisa de campo com trinta especialistas da região metropolitana de São Paulo, investigando-se a os critérios usualmente adotados para a escolha por um determinado modelo de bomba de circulação. Os resultados da pesquisa mostraram que os critérios prevalentes na escolha foram o fato da bomba ser um produto importado, devido a uma percepção de qualidade dos consumidores associada aos fabricantes internacionais, e o fator preço, que normalmente pende a favor dos produtos nacionais, sendo o consumo energético o fator menos levado em consideração nessa escolha.

Este artigo tem duas contribuições científicas, com relação às bombas de circulação utilizadas em Aquariofilia, a proposição da classificação conforme suas vazões (Tabela 7) e de critério de seleção baseado na ecoeficiência (Tabela 9).

Os resultados deste estudo mostraram que na seleção de bombas de baixa e média vazões, os períodos de retorno do investimento são consideravelmente elevados, de modo que a economia de energia proporcionada dificilmente justificaria por si só a escolha pelo modelo mais eficiente, normalmente de maior preço. Para estas vazões, contudo, sugere-se o critério de seleção baseado no maior prazo de garantia do produto, supondo-se que com isto, seja o produto mais durável, visando-se assim a não impactar o meio-ambiente com um produto que venha a falhar em serviço precocemente, além do risco de poder comprometer a vida animal.

Para bombas de circulação de alta vazão, de acordo com as análises deste estudo, o critério de ecoeficiência, materializado no menor consumo de eletricidade para a operação, deveria ser levado em consideração, o que não se comprovou durante pesquisa de campo. Constatou-se a inexistência de uma preocupação em relação à eficiência ou ao consumo de energia elétrica, pela falta de um critério que incorporasse aspectos de ecoeficiência. Esse fato deve-se, provavelmente, à carência de informação sobre os ganhos econômicos que seriam obtidos com o uso de um equipamento mais eficiente, ou quiçá 
devido a uma falta de preocupação/ consciência com a preservação dos recursos energéticos e ambientais (Albuquerque et al., 2019; Silva et al., 2019a; Silva et al., 2019b; Mensah et al., 2019).

Como limitações deste trabalho deve-se destacar, o número limitado de 30 especialistas pesquisados, ao critério de conveniência utilizado de circunscrever-se a amostra analisada à região metropolitana de São Paulo, e possíveis vieses nas respostas, dada a diversidade de entendimentos, por falta de uma padronização.

Outra limitação é que o critério de seleção de bombas de circulação usada em Aquariofilia proposto, não leva em consideração as quantidades de peixes inseridos no sistema, o tipo de filtragem utilizada, o tipo de alimentação, a época do ano e ornamentos existentes no sistema, tal qual nos critérios atuais. Assim propõem-se estudos futuros para avaliar como cada uma destas variáveis impacta no critério de seleção proposto.

Sugere-se também para pesquisas futuras, a ampliação desta pesquisa, estendendo-a para outros estados da federação, de forma a confrontar-se com os resultados deste trabalho.

\section{Agradecimentos}

Os autores agradecem à Coordenação de Aperfeiçoamento do Ensino Superior (CAPES), pela concessão de auxílio e pelo apoio para realização deste trabalho.

\section{Conflitos de interesse}

Os autores declaram não haver conflitos de interesse.

\section{Referências}

Albuquerque, C. C. B.; Maciel, L. R.; Nakamori, S.; Auler, T.; Catapan, A. Panorama da energia eólica sob a perspectiva dos impactos ambientais no Brasil. Revista Brasileira de

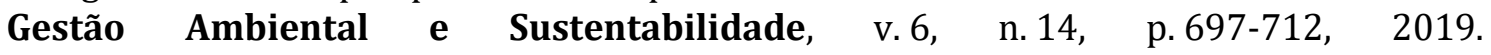
https://doi.org/10.21438/rbgas.061406

ANEEL - Agência Nacional de Energia Elétrica. Postos tarifários. 2020. Disponível em: <http://www.aneel.gov.br/postos-tarifarios>. Acesso em: 04 abr. 2020.

Bertheau, P. Assessing the impact of renewable energy on local development and the Sustainable Development Goals: Insights from a small Philippine Island. Technological Forecasting and Social Change, v. 153, 119919, 2020. https://doi.org/10.1016/ j.techfore.2020.119919

Carino, S.; Mccartan, J.; Barbour, L. The emerging landscape for sustainable food system education: Mapping current higher education opportunities for Australia's future food and nutrition workforce. Journal of Hunger and Environmental Nutrition, v. 15, n. 2, p. 273-294, 2020. https://doi.org/10.1080/19320248.2019.1583621

Coe, C. M.; Araújo, R. C. P. Análise da sustentabilidade da cadeia produtiva de peixes ornamentais na Região Metropolitana de Fortaleza-CE. Anais do 48o Congresso da Sociedade Brasileira de Economia, Administração e Sociologia Rural, Campo Grande, p. 1-21, 2010.

Colpani. Demanda por Aquarismo cresce no Brasil. 2016. Disponível em: <https://www.grupoaguasclaras.com.br/demanda-por-aquarismo-cresce-no-brasil>. Acesso em: 07 mar. 2020.

ELEKTRO. Eficiência energética: fundamentos e aplicações. Campinas: Elektro, 2012. 
FAO - Food and Agriculture Organization. The state of world fisheries and aquaculture: Meeting the sustainable development goals. Roma: FAO, 2018. Disponível em: <http://www.fao.org/state-of-fisheries-aquaculture>. Acesso em: 15 mar. 2020.

Fox, R. W. Introduction to the fluid mechanics. 6. ed. Perdue: John Wiley \& Sons, 2004.

Fox, R. W.; McDonald, A. T.; Pritchard, P. J. Introdução à mecânica dos fluidos. 6. ed. São Paulo: LTC, 2006.

Huppes, G.; Ishikawa, M. Eco-efficiency and its terminology. Journal of Industrial Ecology, v. 9 n. 4, p. 43-46, 2005.

IBGE - Instituto Brasileiro de Geografia e Estatística. Disponível em: <https://www.ibge.gov.br/>. Acesso em: 25 maio 2018.

Maroun, C.; Schaeffer, R. Emulating new policy goals into past successes: Greenhouse gas emissions mitigation as a side effect of biofuels programmes in Brazil. Climate and Development, v. 4, p. 187-198, 2012. https://doi.org/10.1080/17565529.2012.668849

Mensah, J. H. R.; Souza, A. R.; Ribeiro N. S.; Freitas, L. C. F.; Silva, A. T. Y. L.; Marros, R. M. Potencial de aproveitamento energético de biogás através de reatores UASB: estudo de caso em Itaúna, Minas Gerais, Brasil. Revista Brasileira de Gestão Ambiental e Sustentabilidade, v. 6, n. 14, p. 609-621, 2019. https://doi.org/10.21438/rbgas.061402

Minayo, M. C. S.; Deslandes, S. F.; Cruz Neto, O.; Gomes, R. Pesquisa social: teoria, método e criatividade. Petrópolis: Vozes, 2002.

MPA - Ministério da Pesca e Aquicultura. Instrução Normativa MPA no 17, de 11 de agosto de 2014. Dispõe sobre a licença de Empresa que Comercializa Organismos Aquáticos Vivos - ECOAV, no Registro Geral da Atividade Pesqueira - RGP. Disponível em: <https://www1.icmbio.gov.br/cepsul/images/stories/legislacao/Instrucao_normativa/2 014/in_mpa_17_2014_licença_ecoav_rgp.pdf>. Acesso em: 15 mar. 2020.

Ono, E. A.; Kubitza, F. Construção de viveiro e de estruturas hidráulicas para o cultivo de peixes: Parte 4 - 0 reaproveitamento da água e o manejo do solo. Panorama de Aquicultura, v. 13, n. 75, p. 17-27, 2003.

Ono, E. A.; Kubitza, F. Planejamento, seleção das áreas, fontes de água, demanda hídrica e propriedades dos solos. Panorama de Aquicultura, v. 12, n. 72, p. 15-29. 2002.

Pritchard, P. J.; Leylegian, J. C. Fox and McDonald's: Introduction to fluid mechanics. 8. ed. Perdue: John Wiley \& Sons, 2011.

SEBRAE - Serviço Brasileiro de Apoio às Micro e Pequenas Empresas. 2019. Disponível em: <http://www.portalsebrae.com.br>. Acesso em: 07 mar. 2020.

Silva, L. M. B.; Silva, J. P.; Borges, M. A. L. Do global ao contexto nacional: evolução da política ambiental brasileira. Revista Brasileira de Gestão Ambiental e Sustentabilidade, v. 6, n. 14, p. 593-608, 2019a. https://doi.org/10.21438/rbgas.061401

Silva, P. C.; Diniz, R. G.; Ferreira, M. A. A.; Santos, D. J. O.; Santos, G. O. Custos de técnicas sustentáveis e aplicáveis em recuperação de áreas de pastagens. Revista Brasileira de Gestão Ambiental e Sustentabilidade, v. 6, $\quad$ n. 14, p.667-676, 2019. https://doi.org/10.21438/rbgas.061404

Valenti, W. C. A aquicultura brasileira é sustentável? Anais do IV Seminário Internacional de Aquicultura, Maricultura e Pesca, Florianópolis, p. 1-11, 2008.

Valenti, W. C. Aquicultura sustentável. Anais do $12^{\circ}$ Congresso de Zootecnia, Vila Real, Portugal, p.111-118, 2002. 
WCED - World Commission on Environment and Development. Our common future. Oxford: Oxford University Press, 1987.

Wood, E. Collection of coral reef fish for aquaria: Global trade, conservation issues and management strategies. Herefordshire: Marine Conservation Society, 2001.

Yang, H.; Zheng, H.; Liu, H.; Wu, Q. Nonlinear effects of environmental regulation on ecoefficiency under the constraint of land use carbon emissions: Evidence based on a bootstrapping approach and panel threshold model. Journal of Environmental Research andEmplas Public Health, v. 16, n. 10, 1679, 2019. https://doi.org/10.3390/ ijerph16101679 